\title{
Characteristics and Factors Associated with Obesity and Thinness among Children with Developmental Disorders
}

\author{
Keiko Kasagi ${ }^{1}$, Yuko Hayashi², Ryoko Ito ${ }^{3}$ \\ ${ }^{1}$ Department of Community Health, Faculty of Health and Welfare, Prefectural University of Hiroshima, Hiroshima, Japan \\ ${ }^{2}$ Department of Pediatrics, Faculty of Health and Welfare, Prefectural University of Hiroshima, Hiroshima, Japan \\ ${ }^{3}$ Department of Maternal Health, Faculty of Health and Welfare, Prefectural University of Hiroshima, Hiroshima, Japan \\ Email: kasagi@pu-hiroshima.ac.jp
}

How to cite this paper: Kasagi, K., Hayashi, Y. and Ito, R. (2018) Characteristics and Factors Associated with Obesity and Thinness among Children with Developmental Disorders. Open Journal of Nursing, 8, 689-696.

https://doi.org/10.4236/ojn.2018.89051

Received: August 27, 2018

Accepted: September 26, 2018

Published: September 29, 2018

Copyright $\odot 2018$ by authors and Scientific Research Publishing Inc. This work is licensed under the Creative Commons Attribution International License (CC BY 4.0).

http://creativecommons.org/licenses/by/4.0/

(c) (i) Open Access

\begin{abstract}
Obesity in childhood or adolescence has been recognized to be a risk factor for the onset of lifestyle-related diseases, not only in healthy children, but also in children with developmental disorders. Therefore, this study was conducted to examine the characteristics of obesity and thinness as assessed by the body fat percentage among children with developmental disorders during certain growth periods. It was also designed to investigate those factors associated with obesity and thinness based on a lifestyle and behavioral questionnaire. The subjects included 260 children from 5 to 18 years old with developmental disorders. The results of the study showed that a decrease in thinness and increase in obesity with ageing exhibited more noticeable trends among those children with mental retardation. The factors associated with obesity in children with developmental disorders were characterized by the dietary content, eating behaviors, and food preferences particular to such children, as well as low physical activity and a family history of obesity. The results of this study suggested the importance of continuous guidance along with family participation in order to improve obesity among children with developmental disorders, while focusing on the characteristics of certain growth periods.
\end{abstract}

\section{Keywords}

Obesity, Thinness, Children with Developmental Disorders, Lifestyle Factors, Body Fat Percentage

\section{Introduction}

The excessive prevalence of obesity has been a growing concern worldwide in 
children as well as adults. This unhealthy tendency has not shown signs of improvement, but rather, it is steadily getting worse. It is well-known that childhood obesity has a great risk of progression into adult obesity. Most notably, Yokoyama et al. suggested that children with mental retardation exhibited an especially high likelihood for this process [1]. In institutions for children with mental retardation, $23 \%$ of the children were obese, while $39 \%$ of the children with Down syndrome were obese in childhood [2]. It has been reported that lifestyle diseases, such as diabetes mellitus, liver functional impairment, and hyperlipidemia, were recognized in obese children with mental retardation [3]. Moreover, Burkhart et al. suggested that the prevalence of obesity was higher in children with mental retardation than in children without health problems [4], and that it was higher in girls than in boys [5].

When compared with obesity, a low body weight in children with developmental disorders has gained less attention, and there are few reports considering the problem of underweight children with developmental disorders. However, we are aware of the existence of extremely low weight cases among children with developmental disorders.

It is a serious concern that sufficient countermeasures against weight problems in children with developmental disorders have not been performed. This is due in part to the existence of particular difficulties in guiding children with typical cognitive-behavioral characteristics toward proper lifestyle habits related to nutrition and physical activities. Therefore, with regard to obesity and thinness, a more pressing need for countermeasures must be recognized for children with developmental disorders, when compared to children without health problems.

\section{Purpose}

The purpose of this study was to identify the characteristics of obesity and thinness among children with developmental disorders. Additionally, it was designed to investigate the lifestyle and behavioral factors associated with obesity and thinness among these children in order to contribute to the implementation of countermeasures for the improvement of obesity among children with developmental disorders.

\section{Methods}

\subsection{Subjects}

This study covered all children with development disorders who were seen during the summer months of July and August in 2009 in the developmental disorder department of a clinic attached to University " $A$ " in Hiroshima, Japan. Among these, 85 patients with cerebral palsy, Down syndrome, and lalopathy were excluded from the study. The remaining 260 children (207 males and 53 females) participated in the study after their parents signed informed consent forms. The children's ages ranged from five to eighteen years old. In this study, 
the developmental disorders and their subclassifications (autism disorder, mental retardation, attention-deficit/hyperactivity disorder, learning disability) were defined and classified based on the Diagnostic and Statistical Manual of Mental Disorders-Fourth Edition (Revision) guidelines [6]. Table 1 shows the study subjects by sex and age, and their diagnostic classifications are also duplicated in Table 1.

\subsection{Data Collection}

Each subject's height was measured by a trained nurse. Their body weights and body fat percentages were measured by employing a dual frequency body composition analyzer (DC-320; Tanita, Tokyo, Japan). The parents were asked about the daily lives of the children with developmental disorders by using a 35 -item questionnaire, which included their dietary habits, physical activities, behavioral activities, and family histories (Table 2). Each questionnaire item had four possible choices: unlikely, less likely, quite likely, and most likely. The questionnaire used in the present study was taken from the questionnaire used in the guidelines for the management of obesity disease [7].

\subsection{Data Analysis}

While employing a regression analysis with the body fat percentage as a dependent variable, the significance of the relationship between the body fat percentage and each item was examined after it was adjusted for sex and age.

\subsection{Ethical Considerations}

This study was conducted under the approval of the Ethics Review Committee of this university.

\section{Results}

\subsection{Distribution of Obesity and Thinness Based on Body Fat Percentage}

\subsubsection{Obesity and Thinness by Sex and Age}

According to the participants' sex and age, the percentage of obese subjects increased with age in both the boys and girls. In the boys, the percentage of obesity increased from $7 \%$ ( 5 - 8 years old), to $29 \%$ (9 - 12 years old), and 35\% (13 - 18 years old). The girls exhibited a similar obesity increase pattern from 12\% (5 - 8 years old), to $33 \%$ ( 9 - 12 years old), and 43\% (13 - 18 years old). Contrarily, thinness was shown in the younger children at higher percentages. The thin subject percentages decreased from $11 \%$, to $9 \%$, and $5 \%$ in the boys, and from $8 \%$, to $5 \%$, and $0 \%$ in the girls (Figure 1 ).

\subsubsection{Obesity and Thinness by Sex and Diagnostic Classification}

According to the sex and diagnostic classification, those children with mental retardation exhibited a greater tendency toward obesity than the children with other diagnostically classified disorders, with rates of $30.3 \%$ in the boys and 
$42.9 \%$ in the girls. With regard to thinness, those children with attention deficit hyperactivity disorder (ADHD) exhibited higher percentages (12\% and $8.3 \%$ in the boys and girls, respectively), while the children with other diagnostically classified disorders exhibited a rate of 3\% - 7\% (Figure 2).

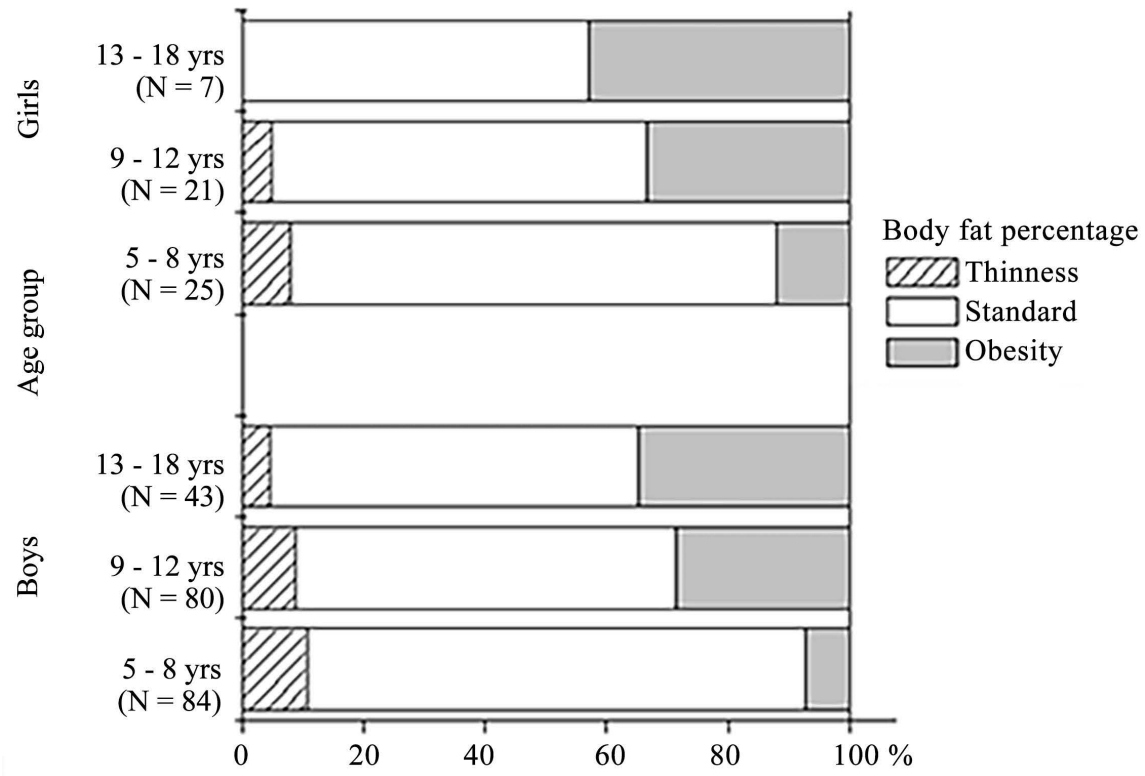

Figure 1. Distribution of obesity and thinness based on the body fat percentage by sex and age.

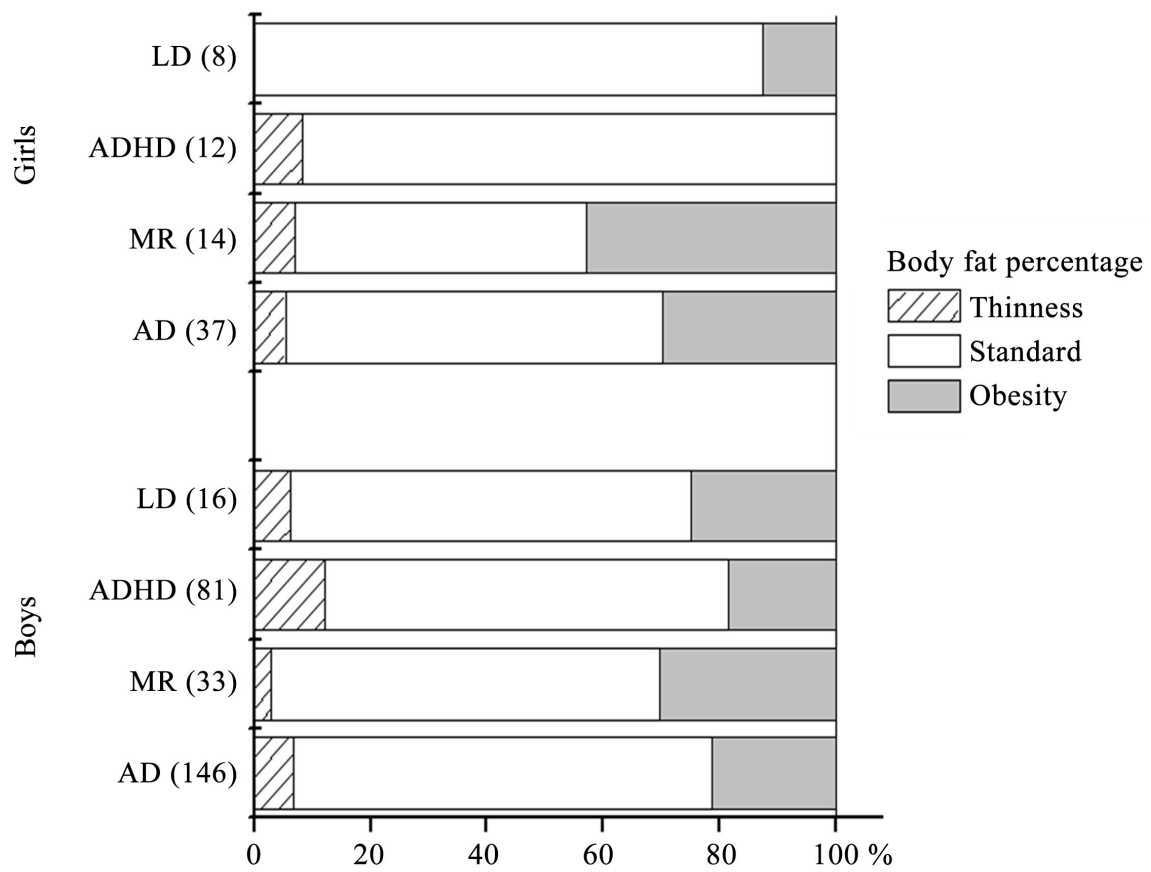

Figure 2. Distribution of obesity and thinness based on the body fat percentage by sex and diagnostic classification. AD: autism disorder, MR: mental retardation, ADHD: attention-deficit/hyperactivity disorder, LD: learning disability. Duplicated case number in parentheses. 
Table 1. Study subjects.

\begin{tabular}{ccccccc}
\hline Sex & Age (yrs.) & Subject number & AD & MR & ADHD & LD \\
\hline \multirow{4}{*}{ Males } & $5-8$ & 84 & $(65)$ & $(11)$ & $(33)$ & $(3)$ \\
& $9-12$ & 80 & $(53)$ & $(15)$ & $(32)$ & $(7)$ \\
& $13-18$ & 43 & $(28)$ & $(7)$ & $(16)$ & $(6)$ \\
Females & $5-8$ & 25 & $(15)$ & $(5)$ & $(8)$ & $(3)$ \\
& $9-12$ & 21 & $(16)$ & $(8)$ & $(4)$ & $(3)$ \\
& $13-18$ & 7 & $(6)$ & $(1)$ & $(0)$ & $(2)$ \\
\hline
\end{tabular}

$\mathrm{AD}$ : autism disorder, $\mathrm{MR}$ : mental retardation, $\mathrm{ADHD}$ : attention-deficit/hyperactivity disorder, $\mathrm{LD}$ : learning disability. Duplicated case number in parentheses.

Table 2. Question items regarding factors associated with the body fat percentage: diet, physical activity, behavior, and family history.

\begin{tabular}{|c|c|c|}
\hline Item & Factor & p-value \\
\hline 1. & Eating at irregular times & 0.084 \\
\hline 2. & Skipping breakfast & 0.078 \\
\hline 3. & Having dinner late & 0.776 \\
\hline 4. & Often having between-meal snacks & $0.002^{\star}$ \\
\hline 5. & Often having late-night snacks & 0.313 \\
\hline 6. & Often eating out & 0.920 \\
\hline 7. & Eating mainly meat & $0.030^{*}$ \\
\hline 8. & Eating less vegetables & $0.019^{*}$ \\
\hline 9. & Often eating fast foods, such as hamburgers & 0.762 \\
\hline 10. & Often eating snack foods or sweetened buns & $0.015^{\star}$ \\
\hline 11. & Preferring fatty foods & $<0.001^{*}$ \\
\hline 12. & Preferring sweet foods & $0.050^{*}$ \\
\hline 13. & Often drinking sweetened beverages & $0.470^{*}$ \\
\hline 14. & Preferring noodles & 0.057 \\
\hline 15. & Eating quickly & $<0.001^{*}$ \\
\hline 16. & Not chewing well & $<0.001^{*}$ \\
\hline 17. & Not being satisfied until having a sense of satiety & $<0.001^{*}$ \\
\hline 18. & Overeating when stressed & $<0.001^{*}$ \\
\hline 19. & Eating when not doing anything & $<0.001^{*}$ \\
\hline 20. & Stuffing mouth when eating & $<0.003^{*}$ \\
\hline 21. & Being a picky eater & 0.681 \\
\hline 22. & Seasoning food heavily & $0.007^{*}$ \\
\hline 23. & Few opportunities to have a meal together with the family & 0.350 \\
\hline 24. & Gaining weight over the holidays & $<0.001^{*}$ \\
\hline 25. & Always having food close at hand & $0.043^{*}$ \\
\hline 26. & Not preferring physical exercise & $<0.001^{*}$ \\
\hline
\end{tabular}




\section{Continued}

\begin{tabular}{lcc}
\hline 27. & Rarely playing outside & $<0.001^{\star}$ \\
28. & Frequently watching TV or playing video games & $0.028^{\star}$ \\
29. & Being prone to constipation & 0.521 \\
30. & Not usually washing hands after coming home from being outside & 0.302 \\
31. & Not brushing teeth regularly & 0.060 \\
32. & Having an uncontrollable desire when wanting something & 0.281 \\
33. & Being impatient when told "tomorrow" or "later" & 0.662 \\
34. & Having overweight family members (siblings, parents, and grandparents) & $0.001^{\star}$ \\
35. & Having a working mother & 0.998 \\
\hline
\end{tabular}

${ }^{*} \mathrm{p}<0.05$ by regression analysis, with sex and age adjustments.

\subsection{Lifestyle and Behavioral Factors Associated with Obesity and Thinness}

Among the 35 items surveyed using the lifestyle and behavioral questionnaire, 19 items were identified as factors that were significantly related to the body fat percentage (Table 2). These significant factors were as follows: dietary pattern (such as having a snack between meals), meat-eating, eating too many snacks or sweetened buns, a poor vegetable intake, food preferences (such as fatty foods or sweets), preferences for deep, salty, and sweet tastes, certain eating behaviors (such as eating quickly), not chewing well, a deep attachment to eating (such as satiety and stuffing one's mouth), overeating due to stress or frustration, eating when there is nothing else to do, a family environment in which food is always on hand, gaining weight due to long holidays, low physical activity (such as a dislike of physical exercise), not playing outside, watching TV, playing video games, and a family history of obesity.

Contrarily, no lifestyle and behavioral factors asked about in the questionnaire were associated with thinness in children with developmental disorders (data not shown).

\section{Discussion}

The results of this study showed that obesity was prevalent in children with mental retardation, which agreed with a previous study conducted in Japan. Nagao reported a significantly higher prevalence of obesity in a school for mentally retarded children than that in the nationwide average of obesity [2]. Our study also determined a higher prevalence of obesity in children with mental retardation than in those with other diagnostically classified disorders, as seen similarly in a report by Naka et al. [3]. One study of adults with mental retardation who were over forty years old [8] also observed a higher proportion of obesity when compared to the healthy adults. Moreover, it has been reported that mental retardation itself induces obesity [9]. Many papers have reported that in children with developmental disorders, females are more likely to be obese than males [2] [5] [10]. 
The present study reported a higher prevalence of thinness among the children with ADHD when compared to those with other diagnostically classified disorders. However, there seemed to be no previous reports that examined the association between ADHD and thinness. Therefore, further investigations are required to confirm these results.

Even if children with developmental disorders have a small appetite during infancy, the problem of low weight is likely to be solved once they are old enough to develop good eating behaviors. However, a tendency toward obesity was observed at around the age of nine (in the upper elementary school grades), when children are deeply attached to eating, with relatively little physical activity. The results of this study suggested a serious likelihood of obesity in both boys and girls with developmental disorders rather than the likelihood of low weight, more generally for girls in puberty or adolescence.

Significant factors influential to the body fat percentage were detected in this study, such as the dietary contents, eating habits, or food preferences particular to children with developmental disorders, as well as weight gain due to long vacations and family environments. These factors must be considered in order to develop health guidance involving family members. One previous study [11] suggested major factors inducing obesity in children with mental retardation, such eating an unbalanced diet, eating quickly, having several servings of food at a meal, poor physical activity habits (such as disliking exercise), and few opportunities and little scope for social activities.

None of the questionnaire items were detected as factors related to thinness. This may partially be due to the small number of cases exhibiting thinness. In addition, the questionnaire was not thorough enough to identify the factors associated with thinness in children with developmental disorders. Further improvements would be needed in order to develop the contents of such a questionnaire.

Based on the present study, obesity seemed to start at around 5 - 6 years old, and it did not seem to ever recover on its own. As such, it is necessary to improve this persistent tendency by focusing on interventions from an early stage, as well as encouraging measures based on the characteristics of each disorder. Moreover, it is essential to cooperate with family members and class teachers, as well as to maintain a long-term support system provided by experts without disruption.

Here, we have reported the results of a series of investigations on obesity in children with developmental disorders [12] [13] [14] [15] [16]. In the future, we will further explore adequate and applicable procedures for health guidance on the improvement of obesity in children with developmental disorders.

\section{Conflicts of Interest}

The authors declare that they have no conflicts of interest with regard to this research. 


\section{References}

[1] Yokoyama, Y. (1989) The Ages at Peak Appearance and Disappearance of Obesity in Mentally Retarded Children. The Journal of the Japan Pediatric Society, 93, 1559-1562.

[2] Nagao, H. (2000) Health Problem and provision for Children with Intellectual Disabilities. Advances in The Medicine on Developmental Disabilities, 12, 25-34.

[3] Naka, Y. and Kotani, H. (2003) A Survey of Obese Child with Intellectual Disabilities in the Kinki Area and a Study of Obese Guidance-First Report. The Journal of Child Health, 62, 17-25.

[4] Burkhart, J.E., Fox, R.A. and Rotatori, A.F. (1985) Obesity of Mentally Retarded Individuals: Prevalence, Characteristics, and Intervention. American Journal of Mental Deficiency, 90, 303-312.

[5] Fox, R. and Rotatori, A.F. (1982) Prevalence of Obesity among Mentally Retarded Adults. American Journal of Mental Deficiency, 87, 228-230.

[6] American Psychiatric Association (2000) Diagnostic and Statistical Manual of Mental Disorders. Washington DC.

[7] Japan Society for the Study of Obesity (2006) Guidelines for the Management of Obesity Disease. Life Science Publishing Co. Ltd., Tokyo.

[8] Ito, J. (2005) Obesity of People with Intellectual Disabilities and Ratio of Health Problem Caused by Obesity. Japanese Journal on Developmental Disabilities, 27, 307-315.

[9] Hara, M., Egawa, K., Nakashita, T., Yamanishi, T. and Shimoda, M. (2001) Intellectual Disability and Obesity. Japanese Journal on Developmental Disabilities, 23, 3-12.

[10] Yamaki, K. (2005) Body Weight Status among Adults with Intellectual Disability in the Community. Mental Retardation, 43, 1-10. https://doi.org/10.1352/0047-6765(2005)43<1:BWSAAW>2.0.CO;2

[11] Naka, Y. and Kotani, H. (2003) A Survey of Obese Child with Intellectual Disabilities in the Kinki Area and a Study of Obese Guidance-Second Report. The Journal of Child Health, 62, 26-33.

[12] Kasagi, K. and Hayashi, Y. (2009) Survey on Frequency of Obesity in Children with Developmental Disorders. Japanese Journal on Developmental Disabilities, 44, 162-163.

[13] Kasagi, K. (2010) Guidance on Obesity Improvement in Children with Developmental Disorders. Japanese Journal on Developmental Disabilities, 45, 308-309.

[14] Kasagi, K. (2009) Examination of Obesity Index of Children with Developmental Disorders-First Report. Journal of Japan Society for the Study of Obesity, 30, 269.

[15] Kasagi, K. (2010) Examination of Obesity Index of Children with Developmental Disorders-Second Report. Journal of Japan Society for the Study of Obesity, 31, 168.

[16] Kasagi, K. (2012) Characteristics from the Frequency Investigation of Thinness and Obesity among Children with Developmental Disorders. Japan. Epidemiological Society, 22, 80. 